\title{
Nonlinear effects and transient response of interband and subgap photoconductivity in polycrystalline sillicon
}

\author{
W. Bock ${ }^{\text {a) }}$ \\ Siemens AG, Zentrale Forschung u. Entwicklung, Onto-Hahn-Ring 6, D-8000 Munich 83, West Germany \\ W. Prett \\ Institut für Angewandte Physik, Universität Regensburg, D-8400 Regensburg, West Germany
}

(Received 17 October 1988; accepted for publication 14 July 1989)

\begin{abstract}
The steady-state photoconductance and the transient photoresponse of boron-doped polycrystalline silicon films have been measured as a function of intensity at photon energies above and below the band gap applying several semiconductor lasers. The intensity dependence of the dc photosignal and the transient behavior are distinctly different for interband and subgap excitation. A kinetic model taking into account grain boundary as well as bulk carrier generation and recombination has been developed describing the observed nonlinear and transient effects consistently. The experimental results yield the density of occupied and unoccupied trap states, absolute values for the optical absorption cross section and thermal capture cross sections and relaxation time constants.
\end{abstract}

\section{NTRODUCTION}

In modern integrated circuit technologies polycrystalline silicon thin films (poly-Si) play an important role. Depending on the kind of deposition various applications of films have been reported. ${ }^{1-3}$ As a first step for three-dimensional integration poly-Si is used for a fabrication of thin film transistors. ${ }^{4}$ An important application of poly-Si fims in microelectronics is its use as gate electrode for field-effect transistors, as local circuit interconnection, and as material for resistors. In modern bipolar transistors technology heavily doped poly-Si films act in two ways as diffusion sources and as highly conductive interconnections to the external transistor contacts. Despite improvement in deposition techniques and post-treating methods, the films have an enhanced resistivity compared to equally nigh-doped monocrystalline silicon at all doping concentrations. ${ }^{5}$ For free carrier concentrations less than about $10^{18} \mathrm{~cm}^{\cdots 3}$ this higher resistivity is generally related to energy states in the band gap, being localized at the so-called grain boundaries, the disordered region between two adjacent grains. These states are caused by the lattice mismatch at the joint of differently orientated grains and act as trapping and recombination centers. By capturing free carriers, a symmetrical depletion zone and an electrostatic potential barrier arises, which impedes the flow of the majority carriers and drastically reduces the carrier mobility. "The total accumulated charge at the grain boundary is given by a two-dimensional trap density being strongly dependent on the specific material parameters. Therefore, much work has been done in evaluating the trap density for differently processed polysilicon films. The applied methods may be distinguished as electro-optical or pure electrical methods. ${ }^{7,8}$ In almost any case, many unknown parameters are assumed involving more or less complex transport mechanisms.

In this study we present an extensive characterization of medium-doped thin poly-Si flms with respect to trap den-

\footnotetext{
${ }^{a}$ Present address: Siemens AG, HL $P$ IS PT, Wernerwerkstrasse 2, D. 8400 Regensburg, West Germany.
}

sity and trap behavior. For this aim we applied a new optical method based on nonlinear photoconductance which allows the investigation of grain boundary and buk effects separately. Applying semiconductor lasers, the intensity of irradiation has been varied over several orders of magnitude. Using lasers with photon energies above and below the silicon band-gap interband photoresponse and subgap photoconductivity have been measured as a function of photon fux density. In addition to these quasi-steady-state measurements of nonlinear photoconductivity, the transient photoresponse of signal rise and decay have been recorded as a function of light intensity. As an important result, both acand dc-photoresponse measurements are necessary to determine absolute values for trap densities together with the optical and thermal cross sections for hole generation and recombination at the grain boundary and in the grains. In previous works, parts of these parameters had to be estimated because of data obtained from different kinds of samples. In particular, of amorphous silicon films.

To our knowledge the observed noninear behavior of photoconductivity for subgap and interband wavelengths has not been reported before.

\section{EXPEPIXENTAL DETALS}

The investigated poly-Si films were prepared by chemical vapor deposition. The flims were deposited at $560^{\circ} \mathrm{C}$ on a 310 mm-thick film of $\mathrm{SiO}_{2}$, which was thermally grown on a lightly p-doped silicon wafer. Due to the low growing temperature, the films initialy had an amporphous structure. The dimensions of the samples are 380 -nm fim thickness and an area of $10 \times 20 \mu \mathrm{m}^{2}$. Doping was carried out by implanting boron ions with a dose of $5.0 \times 10^{13} \mathrm{~cm}^{-2}$ at $40 \mathrm{keV}$. Electrical contacts were formed by an additional boron dose of $5.0 \times 10^{15} \mathrm{~cm}^{-2}$. Finally, the films were annealed and recrystallized at $950^{\circ} \mathrm{C}$ for $8 \mathrm{~min}$. From Hall neasurements the free hole concentration was estimated to be $(5.2 \pm 0.2)$ $\times 10^{17} \mathrm{~cm}^{-3}$. As the doping concentration is rather high, the Hall constant does not have to be corrected to compensate 
for grain boundary effects. Thus about one half of the acceptors are ionized.

Either an AlGaAs semiconductor laser emitting at 830 $\mathrm{nm}$ wavelength or one of two different GaInAs lasers with wavelengths of 1.3 and $1.5 \mu \mathrm{m}$ was used as an irradiation source. The dc photon fux density was controlled by changing the light intensity by use of a current source. To observe photosignal transients a pulse generator and a laser driver circuit were used to modulate the laser current for creating steep rectangular light pulses. The eminted laser light was monitored by calibrated $\mathrm{Si}$ - and Ge-photodiodes to determine photon flux density and light pulse shape. All measurements have been performed at room temperature.

\section{NEXPERIKEMTAL RESULTS}

The results of dc measurements are shown in Fig. 1, where the photoconductance $G$ is given as a function of photon flux density $F$. The three curves belong to the three wavelengths $\lambda_{1}=830 \mathrm{~nm}_{2} \lambda_{2}=1.3 \mu \mathrm{m}$ and $\lambda_{3}=1.5 \mu \mathrm{m}$. While the photoconductance for $\lambda_{2}$ and $\lambda_{3}$ behaves similarly, the photosignal for the shorter wavelength is distinctly different. For interband light $\left(\lambda_{1}\right)$ the signal increases in a sublinear manner like $F^{v}$ with $v$ decreasing from 0.69 to a final value of 0.32 at $F \approx 10^{20} \mathrm{~cm}^{-2} \mathrm{~s}^{-1}$. For higher intensities the increase of the interband photosignal saturates. At the subgap wavelengths $\lambda_{2}$ and $\lambda_{3} G$ increases superinearly for $F \leqslant 10^{18}$ $\mathrm{cm}^{-2} \mathrm{~s}^{-1}$ meaning that $G$ increases faster than $F_{0}{ }^{10}$ For $F$ larger than $10^{19} \mathrm{~cm}^{-2} \mathrm{~s}^{-1}$ the photoconductance shows saturation behavior. At a fixed value of photon fux density the photoconductance increases with decreasing wavelength. The ratio between the photoconductance for $\lambda_{1}$ and $\lambda_{2} \mathrm{de}$ creases with increasing $F$ from two orders of magnitude at $F=10^{17} \mathrm{~cm}^{-2} \mathrm{~s}^{-1}$ to a factor of 2 at $F \geqslant 10^{20} \mathrm{~cm}^{-2} \mathrm{~s}^{-1}$. The fractional change of the current through the sample is about $15 \%$ for the maximum photon fux density.

Figure 2 shows the photosignal transients at the interband wavelength $\lambda_{1}=830 \mathrm{~mm}$. The three different symbols indicate various steady-state excitation intensities at switching on [Fig. 2(a)] and switching off [Fig. 2(b)] the light. Care was taken so that the rising and falling flanks of the corresponding light puise were much steeper $(<350$ ps) than the measured photoresponse transients. Signal rise as

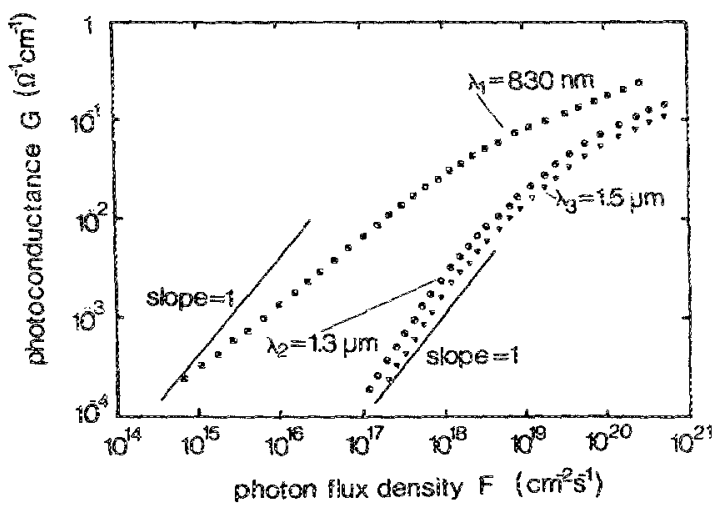

FIG. 1. Photoconductance $G$ as a function of photon fiux density $F$ for interband excitation $\left(\lambda_{1}\right)$ and for two subgap wavejengtins.
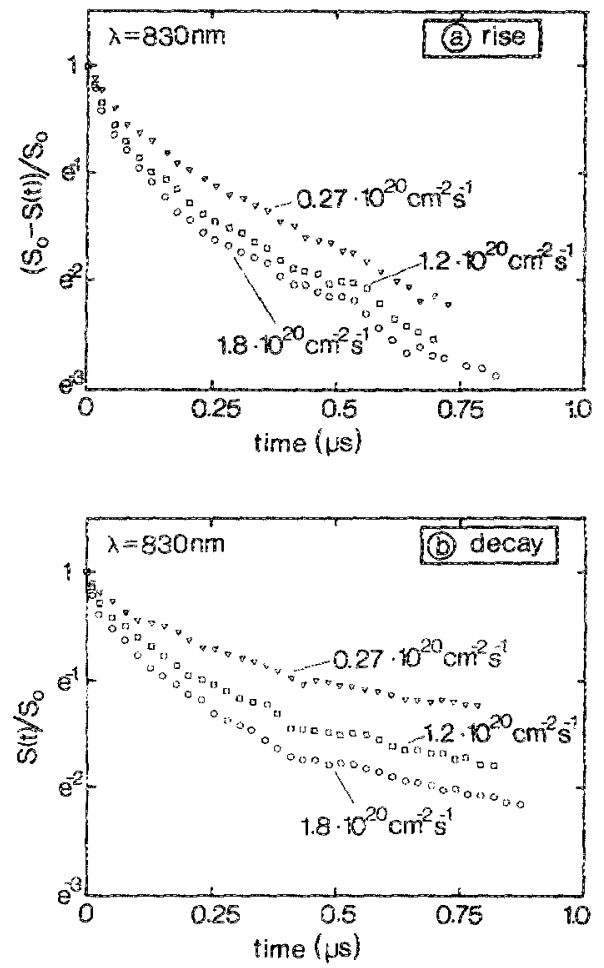

FIG. 2. Rise (a) and decay (b) of the interband photosignal $S(t)$ notmalized to the steady-state photosignal $S_{0}$. The three symbols belong to three different excitation photon fiw densities.

well as signal decay have a nonexponential behavior. Nevertheless if a time-dependent parameter $\tau(t)$ is defined by $\exp [-t / \tau(t)]$ being equal to $S(t) / S_{0}$ for the decay and to $\left[S_{0}-S(t)\right] / S(t)$ for the signal rise, where $S_{0}$ is the initial or firal steady-state photoconductive signal, $\tau(t)$ will increase with time. On a long time scale $(t>0.3 \mu \mathrm{s}) \tau(t)$ is larger for the decay process compared to the signal rise and is generally less sensitive to a variation of $S_{0}$.

Figures $3(a)$ and $3(b)$ display the corresponding photoresponse transients for the subgap excitation. By comparison of Figs. 2 and 3 , the rise and decay of the photosignal seems to be faster for the interband excitation. For 0.2 $\mu s<t<0.5 \mu \mathrm{s} \tau(t)$ is about two times smaller for interband photoconductivity than in the case of subgap excitation.

\section{MODEL}

Current transport in polycrystalline silicon thin films can be described by a thermionic emission model. In this approach the existence of uniform sized grains and in the case of $p$-doped material a shallow simply ionized acceptor with concentration $N_{A}$ is assumed. Figure 4 shows schematically the energy band bending and the trap distribution of one grain and two adjacent grain boundaries in this onedimensional model. Without illumination, localized states at the grain boundaries are charged due to trapped holes down to the equilibrium Fermi energy level $E_{F}$. This charge is compensated by ionized acceptors in a depletion layer on both sides of the boundary. The trapped carriers cause an electrostatic potential barrier $\Phi_{B}$, which controls the dark current density $j_{d}$ according to 

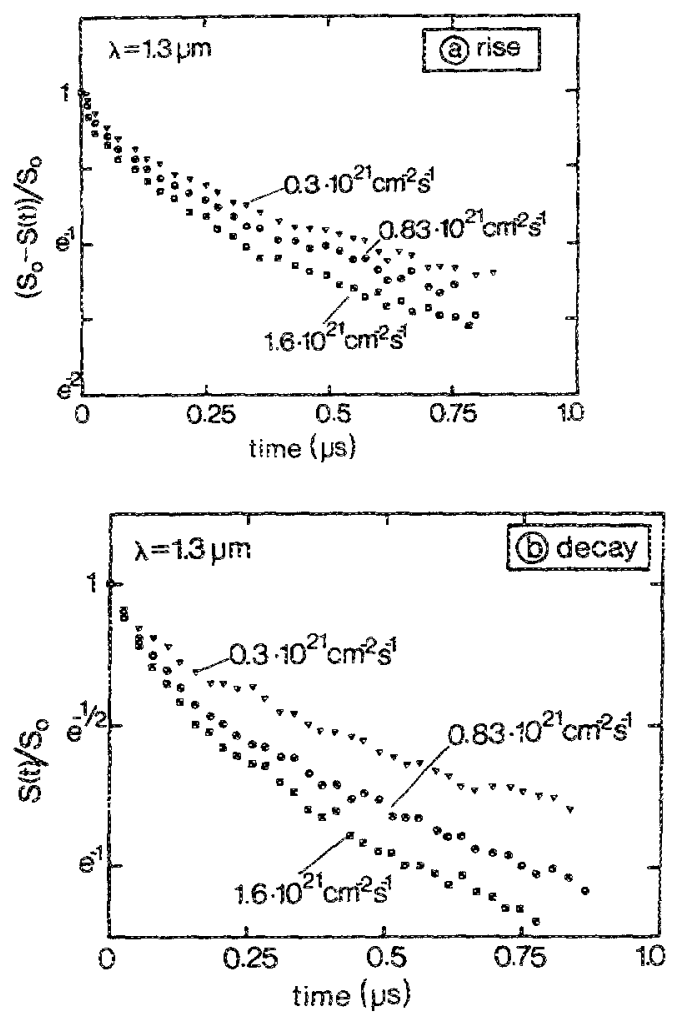

FIG. 3. Photosignal rise (a) and decay (b) at the excitation wavelength $\hat{\lambda}_{2}=1.3 \mu \mathrm{m}$. The symbols and labels have the same meaning as in Fig. 2 .

$$
j_{d} \sim \exp \left[-e\left(\omega_{B}+\xi\right) / k T\right]
$$

(Ref. 7), where $e \xi$ is the potential difference between the Fermi energy level $E_{F}$ and the valence band edge $E_{Y}$. Considering the temperature dependence of $\xi$, the barrier height $\Phi_{B}$ was determined from the temperature-activated dark conductivity to be $\Phi_{B}=73 \mathrm{mV}$. Using the free hole density $p$, the potential $\xi$ follows from Boltzmann distribution to $\xi=-k T / e \ln \left(p / N_{V}\right)=90 \mathrm{mV}$, where $N_{V}$ is the efiective

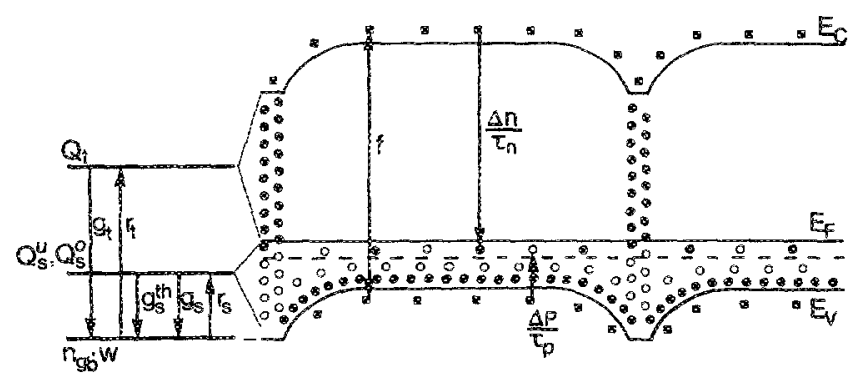

FIG. 4. Scheme of the band bending and trap distribution in the grains and at the grain boundary. The flled circles represent the ionized acceptors at the valence band edge $E_{v}$, the empty circles describe the unoccupied shallow trap states between the Fermi energy level $E_{F}$ (or quasi Fermi energy level $E_{F_{D}}$ ) and $E_{V}$. The circles and squares filled with a cross represent occis. pied deep trap states and free holes, respectively. The minority generation rate is denoted by $f$ the recombination time constants of optically excited electrons $\Delta n$ and holes $\Delta p$ are given by $\tau_{n}$ and $\tau_{p}$. On the left side of the figure the applied three level model with the trap densities $Q_{2}, Q_{s}^{*}$, and $Q$ ? and the gentation and recombination rates for the subgap excitation are shown in correspondence with the trap distribution at the grain boundary. density of states at the valence band edge. At high doping levels and smail grain boundary widths tunneling of holes through the potential barrier has to be taken into account. An estimation of the tunnel current according to Ref. 11 shows that in the case of low bias thermal assisted tunneling is much less important for $p-\mathrm{Si}$ in comparison to e.g., $n$ GaAs, mainly because of the larger effective mass of holes in silicon with respect to electrons in GaAs. Therefore the thermionic emission theory may be used in the present case yielding immediately the photocurrent from Eq. (1):

$$
j_{\mathrm{ph}}=j_{d}\left[\exp \left(\frac{e \Delta \xi}{k T}\right) \exp \left(\frac{e \Delta \Phi_{B}}{k T}\right)-1\right]
$$

where $e \Delta \xi$ is the shift of the quasi Fermi level of holes $E_{F o}$ with respect to $E_{F}$ and $\Delta \Phi_{B}$ is the decrease of barnier height.

\section{A. Interband photoconductivity}

We showed previously, ${ }^{12}$ that subgap photons can excite trapped holes from gap states into the valence band. This effect reduces the barrier height but does not increase the free hole density $p$ appreciably. For an interpretation of the significantly higher photoconductance at the interband wavelength the creation of free electrons and holes in the grains must be taken into account. The sublinear intensity dependence of the interband photoconductivity which was observed over more than three orders of magnitude of intensity and the saturation of the signal at high power levels cannot be explained by simple two level saturation modeis. ${ }^{10}$ On the contrary, a distribution of trapping states $P_{i}(E)$ originating from band-tail states at the valence band in the grains has to be taken into account. The fact that the density of states in polysilicon is smeared out into the forbidden gap of the bulk semiconductor, as it is well known in disordered semiconductors, led to good agreement between theory and experimental results for the electrical characteristics of polysilicon, even in not heavily doped materials. ${ }^{13,14}$

In highly doped polysilicon the minority carrier lifetime is smaller than $1 \mathrm{~ns}^{15}$ so that in $p$-doped samples the thermal capture rate for electrons is much greater than for holes. The reason for that might be the effective recombination at the grain boundary as well as at the band-tail states in the bulk. This means that the concentration of optical-excited free holes $\Delta p$ is distinctly higher than the concentration of minorities $\Delta n$. Therefore, interband light increases $p$ by the optically generated hole density $\Delta p$, which is formally described by the shift of the quasi Fermi level $e \Delta \xi$.

$$
\Delta p=p[\exp (e \Delta \xi / k T)-1] \text {. }
$$

On the other hand, $\Delta p$ is equal to the product of the lifetime of holes $\tau_{p}$ with the total generation rate $f$ :

$$
\Delta p=f \tau_{p} ; \quad 1 / \tau_{p}=v_{\mathrm{kn}} \sigma_{p}^{\mathrm{th}} N_{r} .
$$

Thereby the inverse of $\tau_{p}$ is given by the product of the thermal hole velocity $v_{\mathrm{ih}}$ and the majority capture rate $\sigma_{p}^{\text {th }}$ with the density of recombination centers $N_{r}$. For the investigated thin flms $f$ is to a good approximation equal to $F \alpha$ where $\alpha=5 \times 10^{3} \mathrm{~cm}^{-1}$ is the absorption constant at $830 \mathrm{~nm}$. With the shift of $E_{F p}$ downwards more and more trapping states 
are converted to recombination states, because after switch ing of the light most of the holes trapped in the levels between $E_{p}$ and $E_{F p}$ will recombine with electrons. ${ }^{10} \mathrm{As} N_{r}$ increases the lifetime $\tau_{p}$ decreases having the observable result of $\Delta \xi \propto \Delta p \propto F^{v}$, i.e., a sublinear intensity dependence of the photosignal. To a good approximation, the density of recombination centers $N_{r}$ is given by the number of $P_{i}$ states lying between the Fermi level $E_{F}$ and the nonequilibrium quasi Fermi level $E_{F p}$ (Ref. 10):

$$
N_{r}=\int_{E_{F p}}^{E_{F}} p_{p}(E) d E .
$$

On the other hand, the density of recombination centers can be expressed as a function of $\Delta \xi$ by using Eqs. (3) and (4):

$$
N_{r}=f k T / v_{t s} \sigma_{p}^{\text {th }} p e \Delta \xi \text {. }
$$

Thereby the linear approximation of Eq. (3) was used because up to the maximum apphed photon fux density, $e \Delta \xi$ is still smaller than $k T$. The density of the irapping states $P_{t}(E)$ follows by differentiation:

$$
P_{i}(E)=\frac{\partial}{\partial(e \Delta \xi)} \frac{f k T}{v_{\mathrm{th}} \sigma_{p}^{\mathrm{th} p e \Delta \xi}} .
$$

The generation rate $f$ is correlated with $\Delta \xi$ by the exponent $v$ characterizing the sublinear increase of the photocurrent with respect to $F$. The experiments show a gradual decrease of $v$ from 0.69 to 0.32 between minimal and maximum light intensity. For locally constant values of $v$, the following proportionality holds:

$$
P_{t}(E) \sim \frac{\partial}{\partial(\Delta \xi)}\left(\frac{(\Delta \xi)^{1 / \nu}}{\Delta \xi}\right) \sim \Delta \xi^{(1 / v-2)} \text {. }
$$

This means that the variation of the trapping state density changes from a decay behavior to an increase with greater distance to $E_{F}$.

Figure 5 shows $\Delta \xi$ as a function of photon flux density $F$ determined from the measured photoconductance $G$ by use

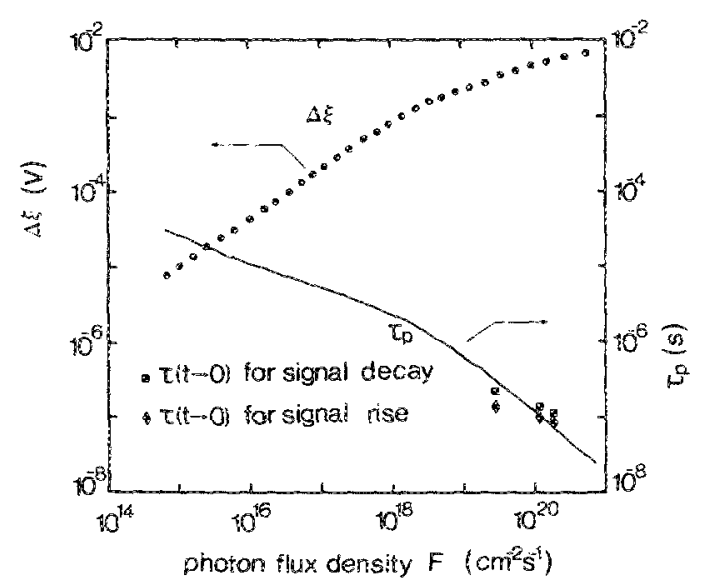

FIG. 5. The potential difference $\Delta \xi$ between the quasi Fermi energy level and the Fermi energy level is drawn as a function of photon fux density $F$. According to the applied theory the majority carrier lifetime $r_{p}$ is shown in relation to $F$. The filled squares and facers give the measured time constants for the decay and rise of the interband photosignai $3(t)$ for $t$ being smaller than $0.25 \mu$. of Eq. (2) and the assumption that $\Delta \Phi_{B} \leqslant \Delta \xi$. This approximation is valid up to about $F \leqslant 10^{20} \mathrm{~cm}^{-2} \mathrm{~s}^{-1}$, where the subgap photosignal is much smaller than the signal for interband excitation. For $F \approx 10^{20} \mathrm{~cm}^{-2} \mathrm{~s}^{-1}$ A $\xi$ is still at least as large as $\Delta \Phi_{B}$. The experimentally determined photocurrent yidds $e\left(\Delta \xi+\Delta \phi_{B}\right) \geqslant 7$ meV while $\Delta \xi$ may be obtained from Eq. (4) and the experimental results for $f$ and $\tau_{p}$ giving $e \Delta \xi \gtrsim 4$ meV. Thus, even in this case, the photocurrent for interband excitation is determined by the majority carrier density.

From Fig. 5 it can be seen that $e A \xi$ is about 1 meV when $v$ becomes less than 0.5 corresponding to $(1 / v-2)>0$. This energy is much smaller than $k T$, so that the decay of the trap density within this narrow energy distance has no physical significance. The larger extent of the energy region chatacterizing an increase of $P_{8}(E)$ allows the interpretation of the trap states as part of the band-tail states at the valence band, which obviously are present in polycrystaline silicon, even if it is not extremely highly doped. Therefore, it is not justified to simply characterize polysilicon as monocrystalline grains connected by grain boundaries. The grains themselves contain a lot of impurity and defect states which are the reason for potential fluctuations and which cause bandtail states.

Beneath the recombination of the minorities through the band-fail states, the way across the grain boundary states is also an important recombination channel. But if the capture rate of holes at the grain boundary is most important, the decay of the photosignal will show a very fast signal transient component to be in accordance with minority lifetimes of less than 1 ns. $^{15}$ As the experiments do not reproduce this fast time constant, it is justified to take the recombination through the band-tail in the grain as the dominant recombination channel.

In Fig. 5, $\tau_{p}$ derived according to Eas. (3) and (4) is shown as a function of $F$. The lifetime $\tau_{p}$ decreases drasticalIy with the growing excitation rate and becomes smaller than 0.1 us for $F \geqslant 10^{20} \mathrm{~cm}^{-2} \mathrm{~s}^{-1}$. The symbols in Fig. 5 correspond to the time-uependent parameter $\tau(t)$ for $t<0.25 \mu s$ from the photosignal transients of Fig. 2. The appropriate values of Fare the initial and final steady-state fux density in Fig. 2. Keeping in mind that $r(t)$ and $\tau_{p}$ result from two different kinds of experiments namely a de and ac method without application of any fiting procedure, the agreenent between the two results is good. In the following $\tau_{p}$ $=(0.12 \pm 0.04) \mu \mathrm{s}$ at $F=10^{20} \mathrm{~cm}^{-2} \mathrm{~s}^{-1}$ will be used.

\section{Subgap photoconductivity}

In the discussion of interband photoconductance effects, the barrier height decrease at the grain boundary $\Delta \bar{\Phi}_{B}$ is assumed to be much smaller than the shift of the quasi Fermi potential $\Delta \xi$. At the subgap wavelengths only $\Delta \Phi_{B}$ has to be taken into account, because additional free holes are created only by excitation out of grain boundary traps, which are localized in a rather narrow region $w_{\mathrm{gh}} \approx 1 \mathrm{~nm}$ (Ref. 16) compared to the large grains with an average width of about $100 \mathrm{~nm}$. As we have shown elsewhere, ${ }^{12}$ at least a three-level generation-recombination model is neces- 
sary to describe the observed superlinear relation between signal and photon flux density and the saturation behavior. By restriction to de measurements, only the ratio of the optical to the thermal cross section could be determined. With the results of the subgap photosignal transients, it is possible to extract the absolute values of the cross sections and to reduce the number of fitting parameters. On the left of Fig. 4 the traps and holes at the grain boundary are related to three levels characterized by different state and carrier densities and various transfer rates for holes between these levels. The energetically lowest level i contains all optically excitable donorlike trap states down to the Fermi energy level. In level 2 the holes trapped in the gap states of the grain boundary which correspond to the $P_{t}$ states of the bulk are accumulated. Level 2 is the extension of the band-tail states from the grains into the grain boundaries. Level 3 is the valence band edge at the grain boundary. It is occupied with a two-dimensional hole density $n_{\mathrm{gb}} w_{\mathrm{g} b}$ :

$$
n_{\mathrm{gb}}=p \exp \left(-e \Phi_{B} / k T\right) \text {. }
$$

Without illumination level 1 is totally occupied with a density $\ell_{t}$. Level 2 is partly filled with holes of density $Q_{s}^{0}$ and has an unoccupied density $Q_{s}^{*}$. The decrease of charge density $Q_{t}$ is determined by optical excitation $g_{\varepsilon}$ and thermal recombination $r_{t}$ :

$$
\begin{aligned}
& g_{t}=k \sigma_{t}^{\kappa p t} F\left(Q_{t}-\Delta Q_{t}\right), \\
& r_{i}=v_{t h} \sigma_{t}^{\text {th }} n_{g^{b}} \exp \left(e \Delta \Phi_{B} / k T\right) \Delta Q_{t} .
\end{aligned}
$$

$\Delta Q_{t}$ stands for the density of optical depleted deep trap states, $\sigma_{z}^{\text {opt }}$ and $\sigma_{r}^{\text {th }}$ are the optical and the thermal cross sections and $v_{\mathrm{th}}$ is the thermal velocity. The factor $k$ denotes the ratio between the polysilicon film thickness and the grain boundary width $w_{\mathrm{g}}$. As level 2 lies closer to the valence band additionally to the optical excitation rate $g_{s}$ and to the thermal recombination $r_{s}$, the thermal excitation rate $g_{c h}$ has to be taken into account:

$$
\begin{aligned}
& g_{s}=k \sigma_{s}^{\text {cpt }} F\left(Q_{s}^{\circ}+\Delta Q_{s}\right), \\
& r_{s}=v_{\mathrm{th}} \sigma_{s}^{\text {th }} n_{\mathrm{gb}} \exp \left(e \Delta \Phi_{B} / k T\right)\left(Q_{s}^{u}-\Delta Q_{s}\right), \\
& g_{\mathrm{th}}=e_{s}^{\text {th }}\left(Q_{s}^{0}+\Delta Q_{s}\right) .
\end{aligned}
$$

$\Delta Q_{s}$ denotes an optically induced increase of the charge density $Q_{s}^{0}$ and $e_{s}^{\text {th }}$ is the thermal emission rate into the valence band. The total decrease of charge density at the grain boundary under illumination $\Delta Q$ is the difference of $\Delta Q_{t}$ and $\Delta Q_{s}$ :

$$
\Delta Q=\Delta Q_{t}-\Delta Q_{s}
$$

In the parabolic band bending modei, ${ }^{6}$ the barrier height $\Phi_{B}$ is related to the local charge density according to

$$
\Phi_{B}=e\left(Q_{i}+Q_{s}^{o}+Q_{\mathrm{ne}}\right)^{2} / 8 \epsilon \epsilon_{0} N_{A},
$$

where $\epsilon_{0}$ is the vacuum permeability and $\epsilon$ is the dielectric constant of silicon. $Q_{\mathrm{nc}}$ is the density of traps being not optical excitable. In the experiments the photocurrent $j_{\mathrm{ph}}$ reaches at maximum $F$ about $20 \%$ of the amount of the dark current, so that the estimation $\Delta \Phi_{E}<k T / e=25 \mathrm{meV}<73$ $\mathrm{meV}=\Phi_{B}$ is valid according to Eq. (2). In this limit Eq. (13) gives a linear dependence of $\Delta \Phi_{B B}$ vs $\Delta Q$ :

$$
\Delta \Phi_{B}=\sqrt{e \Phi_{B} / 2 \epsilon \epsilon_{0} N_{A}} \Delta Q .
$$

The rate equations for $\Delta Q_{t}$ and $\Delta Q_{s}$ of the three level model are

$$
\begin{aligned}
& \frac{d \Delta Q_{t}}{d t}=g_{t}-r_{i}, \\
& \frac{d \Delta Q_{s}}{d t}=-g_{s}-g_{\text {th }}+r_{i} .
\end{aligned}
$$

These two coupled nonlinear ordinary differential equations give an implicit expression for $\Delta \Phi_{s}$ as a function of photon fux density $F$. In the Appendix the equations are further simplified in the case of detailed balance and steady state using the assumptins denoted above. The resuiting two coupled quadratical equations cannot be solved or linearized without losing physical significance. Together with Eq. (14) the equations can oniy be treated numerically to evaluate $\Delta \Phi_{B}$ as a function of $F$ with the densities $Q_{t}, Q_{s}^{0}$, and $Q_{s}^{u}$ as fitting parameters. The unknown ratios of the optical to the thermal cross sections $\sigma_{t}^{\text {opt }} / \sigma_{t}^{\text {th }}$ and $\sigma_{s}^{\text {opt }} / \sigma_{s}^{\text {th }}$ are given by evaluation of the time-dependent equations. As level 2 lies much closer to the band edge than level 1 , the thermal capture rate into shallow traps will be much higher than trapping into the deep levels. Therefore $\sigma_{s}^{\text {th }}$ is larger than $\sigma_{t}^{\text {th }}$ having the result that the decay process is on the long time scale dominated by the recombination into level 1 . This makes a separate solution of Eq. (15a) with the assumption $\Delta Q_{s} \leqslant \Delta Q_{\text {t }}$ worthwhile. At high exciation intensity immediately after switching the light off, $\Delta Q_{t}(t)$ will not change appreciably but $\Delta Q_{s}(t)$ will decrease rapidly. In the light switch on process $\Delta Q_{s}$ is initially much smaller than $\Delta Q_{t}$ and $\mathrm{Eq} .(15 \mathrm{a})$ can again be discussed analytically with the assumption $\Delta Q_{s}$ $\ll \Delta Q_{t}$. These three approximations are further evaluated in the Appendix and, as is shown there, three time constants for comparison with experimental are extractable:

$$
\begin{aligned}
\tau_{s}^{\mathrm{dec}}= & {\left[v_{\mathrm{th}} \sigma_{s}^{\text {th }} n_{\mathrm{gb}}\left(1+d Q_{s}^{u}+Q_{s}^{u} / Q_{s}^{0}\right)\right]^{-1}, } \\
\tau_{t}^{\mathrm{att}}= & \left(v_{\mathrm{th}} \sigma_{t}^{\mathrm{th}} n_{\mathrm{gb}}\right)^{-1} \\
\tau_{t}^{\mathrm{att}}= & {\left[\left(v_{\mathrm{th}} \sigma_{t}^{\text {th }} n_{\mathrm{gb}}\right)^{2}+\left(\sigma_{t}^{\mathrm{th}} k F_{0}\right)^{2}\right.} \\
& \left.+2 v_{\mathrm{th}} \sigma_{t}^{\mathrm{th}} n_{\mathrm{gb}} k \sigma_{t}^{\text {spt }} F_{0}\left(1+2 d Q_{t}\right)\right]^{-2},
\end{aligned}
$$

where

$$
d=(e / k T) \sqrt{e \Phi_{B} / 2 \epsilon \epsilon_{0} N_{A}} .
$$

$\tau_{s}^{\text {dec }}$ is the lifetime for recombination into the shallow states and corresponds to the time constant as it is found in the transients for interband excitation. The time constant $\tau_{t}^{\text {dec }}$ belongs to the signal decay on the long time scale $(t \geqslant 0.3 \mu \mathrm{s})$ in Fig. 3(b). The signal rise in Fig. 3(a) at the subgap wavelength of $1.3 \mu \mathrm{m}$ allows the extraction of $\tau_{t}^{\text {tt }}$ for a fixed photon flux density $F_{0}$.

\section{DISCuSSION}

The values found for the three time constants $\tau_{s}^{\mathrm{dec}}, \tau_{t}^{\mathrm{dec}}$, and $\tau_{t}^{\text {att }}$ are summarized in Table II. Assuming that $\sigma_{t}^{\text {opt }}$ $\approx \sigma_{s}^{\text {opt }}$ and using $\mathrm{Eq}$. (16) to evaluate the cross sections as a function of the trap densities, $\Delta \Phi_{B}$ is fitted to the results of measurement at the wavelengths $\lambda=1.3 \mu \mathrm{m}$ and $\lambda=1.5$ 


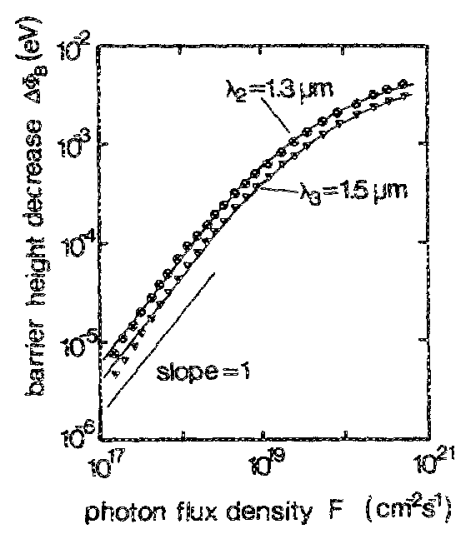

FIG. 6. Decrease of the barrier height $\Delta{ }_{B}$ derived from the neasured photoconductance vs the pinoton fiux density $F$. The symbols give the points of measurement for the two wavelengths, the continuously drawn curves give the results of the fitting procedure for the trap densities.

$\mu \mathrm{m}$. The fit is shown in Fig. 6. The agreement with the experimental results is good for photon fiux densities larger than $10^{18} \mathrm{~cm}^{-2} \mathrm{~s}^{-1}$. The model does not reproduce the superlinear behavior of the photosignal for lowest intensities. This might be due to the relatively large steady-state photon fux densiuies $F 10^{20} \mathrm{~cm}^{-2} \mathrm{~s}^{-1}$ being applied for the measurement of the photosignal rransients. The fitted rap densities and the cross sections given by use of the measured time constants are summarized in Table 1 . To test the self-consistency of the applied model, the rate equations [Eq. (15)] are solved numerically putting in the experimental time constants as well as the trap densities which originate in the last analysis from dc measurements. In Fig. 7 the decay of the subgap photosignal and the numerical results are compared. By consideration of the complexity of the model and the different types of experiments the agreement is surprisingly good despite of quantitative differences. The numerical values for the thermal cross sections in Table I show that the recombination into the shallower states is significantly enhanced. The ratio of $\sigma_{s}^{\text {th }} / \sigma_{i}^{\text {th }}$ especially is 5 and it is therefore much greater than for our samples. For the de photosignal, it causes a larger extent of the saturation region compared to a simple two level model. The absolute values of the themal

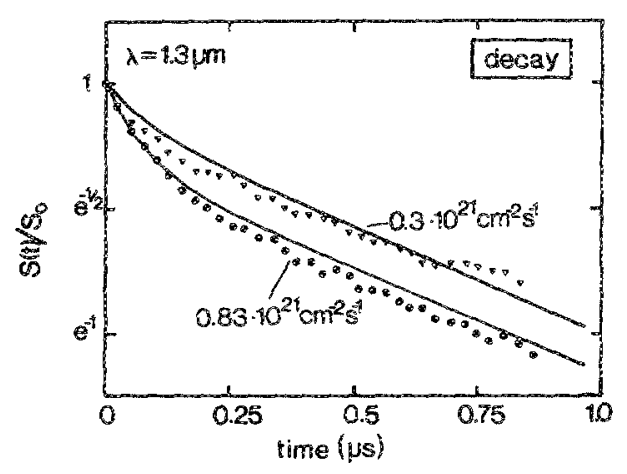

FIG. 7. The measured decay of the subgap photosignal is compared to the numerical solution of the rate equations, which is established by using the time constants and the results for the trap densities.
TABLE 1. The fitted trap densities $Q_{1}, Q_{8}^{0}, Q_{s}^{u}$ and $Q_{n e}$ for subgap excitation at the wavelengths $\lambda_{1}=1.3 \mu \mathrm{m}$ and $\lambda_{3}=1.5 \mu \mathrm{m}$.

\begin{tabular}{ccc}
\hline Wavelength & \multicolumn{1}{c}{$1.3 \mu \mathrm{m}$} & $1.5 \mu \mathrm{m}$ \\
\hline$Q_{\mathrm{r}}$ & $3.6 \times 10^{11} \mathrm{~cm}^{-2}$ & $2.9 \times 10^{11} \mathrm{~cm}^{-2}$ \\
$Q_{s}^{0}$ & $1.6 \times 10^{11} \mathrm{~cm}^{-2}$ & $1.5 \times 10^{11} \mathrm{~cm}^{-2}$ \\
$Q_{s}^{2}$ & $1.1 \times 10^{11} \mathrm{~cm}^{-2}$ & $9.8 \times 10^{10} \mathrm{~cm}^{-2}$ \\
$Q_{m e}$ & $1.75 \times 10^{12} \mathrm{~cm}^{-2}$ & $1.84 \times 10^{12} \mathrm{~cm}^{-2}$ \\
\hline
\end{tabular}

cross sections are somewhat between literature data ranging from $10^{-18} \mathrm{~cm}^{2}$ to $5 \times 10^{-16} \mathrm{~cm}^{2}{ }^{14,17}$ The absolute values of the optical cross section depends on the width of the grain boundary which is not exactly known. For a width of $w_{\mathrm{gb}} \approx 1$ nm $\left(\right.$ Ref. 16) $\sigma_{s, 2}^{\text {opt }}=3.2 \times 10^{-17} \mathrm{~cm}^{2}$ is somewhat smaller than the reported value of $1.2 \times 10^{-16} \mathrm{~cm}^{2}$ for a dangling bond state in amorphous silicon, but is of the same order of magnitude. ${ }^{18}$ The numerical results for the trap densities in Table l show that only one-fourth of the cotal concentration is optically excitable. Therefore the optical cross section would be smaller, if it is taken as an average for the total trap density.

The high value of $Q_{m e}$ might further be the reason for the superlinear behavior of the photosignal at low photon fux densities. Optically depleted states could be reoccupied by a rearrangement of carners being initially not excitable. This causes an intersity dependence of $Q_{i}$ which is not accounted for in our model. From the ratios of $Q_{t}$ to $Q_{s}^{0}$ and $Q_{s}^{u}$ we conclude that the trap density in the middle of the band gap of the grain boundary is not much smaller than the density in the vicinity of the band edge in contrast to the $V$-shaped density of states as it is found by other authors for samples with less impurity concentrations. ${ }^{8}$ The lower value of $Q_{\imath}$ at $1.5 \mathrm{~mm}$ wavelength compared to $\lambda_{2}=1.3 \mu \mathrm{m}$ originates from the larger extent of the region of unexcitabie trap states near the conduction band due to the smaller photon energy.

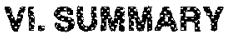

In summary we present a model for nonlinear photoconductance in medium $p$-doped polycrystalline silicon thin flims. Grain boundary effects are investigated by optical excitation with subgap light and bulk effects by irradiation with interband light. Experimentally the photoconductance of the samples was measured over a wide range of photon fux density for three different wavelengths and the rise and decay times of the photosignal were determined as a function of intensity. A model for the photoconduction in polycrys-

TABLE II. Experimental time constants for the subgap photosignai rise $\tau_{t}^{\text {atr }}$ and for the recombination into the shallow trapping states fder and into tize deep states $\tau_{t}^{\text {des }}$. The optical cross section $\sigma_{s, t}^{\text {ogra }}$ and the thermal cross sections $\sigma_{s}^{\text {in }}$ and $\sigma_{1}^{\text {th }}$ are derived by use of the time constants and the trap densities.

\begin{tabular}{ll}
\hline$\tau_{t}^{\mathrm{atg}}=0.18 \mu \mathrm{s}$ & $\sigma_{s t}^{\mathrm{spt}}=3.2 \times 10^{-17} \mathrm{~cm}^{2}$ \\
$\tau_{t}^{\text {dec }}=1.23 \mu \mathrm{s}$ & $\sigma_{t}^{\text {th }}=3.01 \times 10^{-18} \mathrm{~cm}^{2}$ \\
$\tau_{\mathrm{s}}^{\mathrm{des}}=0.12 \mu \mathrm{s}$ & $\sigma_{\mathrm{s}}^{\mathrm{th}}=1.44 \times 10^{-17} \mathrm{~cm}^{2}$ \\
\hline
\end{tabular}


talline silicon was developed which allows the determination of the absolute values of the density of majority carrier traps at the grain boundaries and in the grains. Additionally the optical and themal cross sections for the excitation and recombination of holes between the rrap states and the valence band states could be found.

\section{APPENDIX}

For a further evaluation of the rate equations [Eq. (15) ] the definition of some abbreviations is useful:

$$
\begin{aligned}
& b_{t}=v_{\mathrm{th}} \sigma_{t}^{\mathrm{th}} n_{\mathrm{gbs}} \\
& b_{s}=v_{\mathrm{th}} \sigma_{s}^{\mathrm{th}} n_{\mathrm{gb}}, \\
& a_{t}=k \sigma_{t}^{\mathrm{opt} / b_{t},} \\
& a_{s}=k \sigma_{s}^{\mathrm{opt}} / b_{s}, \\
& d=(e / k T) \sqrt{e \Phi_{B} / 2 e \epsilon_{0} N_{A}}, \\
& C=1+d Q_{s}^{u}+Q_{s}^{u} / Q_{s}^{o} .
\end{aligned}
$$

The assumption $\Delta \Phi_{B} \leqslant k T / e$ allows an approximation of the exponential expression in Eq. (15):

$$
\exp \left(\frac{e \Delta \Phi_{B}}{k T}\right) \approx 1+\frac{e \Delta \Phi_{B}}{k T}=1+d\left(\Delta Q_{t}-\Delta Q_{s}\right)
$$

where Eqs. (12) and (14) are applied. By insertion of Eqs. (10) and (11) into Eq. (15) along with the abbreviations (A1) it follows:

$$
\begin{aligned}
\frac{\partial Q_{t}}{\partial t}= & \sigma_{i}^{o p t} k F\left(Q_{t}-\Delta Q_{t}\right)-b_{t}\left[1+d\left(\Delta Q_{t}-\Delta Q_{s}\right)\right] \Delta Q_{t}, \\
\frac{\partial Q_{s}}{\partial t}= & -\left(\sigma_{s}^{o n} k F+e_{s}^{t h}\right)\left(Q_{s}^{o}+\Delta Q_{s}\right) \\
& +b_{s}\left[1+d\left(\Delta Q_{t}-\Delta Q_{s}\right)\right]\left(Q_{s}^{u}-\Delta Q_{s}\right) .
\end{aligned}
$$

The thermal emission rate $e_{s}^{\text {ith }}$ is derived by taking into account that $\Delta Q_{s}=0$ for $F=0$.

$$
e_{s}^{\text {th }}=b_{s} Q_{s}^{u} / Q_{s}^{0}
$$

Using this identity the equations (A3) are rewritten:

$$
\begin{aligned}
\frac{\partial Q_{t}}{\partial t}= & \sigma_{t}^{\mathrm{opt}} k F Q_{t}-\left(\sigma_{t}^{\mathrm{opt}} k F+b_{t}-b_{t} d \Delta Q_{s}\right) \\
& \times \Delta Q_{t}-b_{t} d\left(\Delta Q_{t}\right)^{2} \\
\frac{\partial Q_{s}}{\partial t}= & b_{s} d Q_{s}^{u} \Delta Q_{t}-\sigma_{s}^{\mathrm{opt}} k F Q_{s}^{o} \\
& -\left(\sigma_{s}^{\mathrm{opt}} k F+b_{s} C+b_{s} d \Delta Q_{t}\right) \Delta Q_{s}+b_{s} d\left(\Delta Q_{s}\right)^{2}
\end{aligned}
$$

\section{Detalled balanco}

In the case of detailed balance, the derivations of Eqs. (A4) and (A5) are zero. By division with $b$, and $b_{s}$ it follows:

$$
\begin{aligned}
& a_{t} F Q_{t}-\left(a_{t} F+1+d \Delta Q_{s}\right) \Delta Q_{t}-d\left(\Delta Q_{t}\right)^{2}=0 \\
& d Q_{s}^{u} \Delta Q_{t}-a_{s} F Q_{s}^{0}-\left(a_{s} F+C+d \Delta Q_{t}\right) \Delta Q_{s} \\
& \quad+d\left(\Delta Q_{s}\right)^{2}=0 .
\end{aligned}
$$

The two coupled quadratical equations cannot be solved analytically. For the applied numerical procedure the values for $a_{t}$ and $a_{s}$ are taken from the time constants for signal rise and decay (see below). The difference of $\triangle Q_{t}$ and $\Delta Q$. which is proportional to $\Delta \Phi_{B}$, is fitted to the barrier height decrease given by the experiment, while the trap densities $Q_{t}$, $Q_{s}^{\circ}$ and $Q_{s}^{u}$ are systematically varied for best agreement.

\section{Solution for time dependent equations}

With the assumption $\Delta Q_{s} \leqslant \Delta Q_{t}$ the differential equation (A 4 ) can be solved analytically. For the recombination process, the photon fux density $F$ is zero and the first equation of (A3) is simplified to

$$
\begin{aligned}
\frac{\partial Q_{t}}{\partial t} & =-b_{t}\left[\Delta Q_{t}+d \Delta Q_{t}\left(\Delta Q_{t}-\Delta Q_{s}\right)\right] \\
& \approx-b_{t} \Delta Q_{t}\left(1+d \Delta Q_{t}\right) .
\end{aligned}
$$

This differential equation is of the form:

$$
\frac{\partial y}{\partial x}=\alpha y^{2}+\beta y
$$

which has the general solution

$$
y(x)=-(\beta / \alpha) \exp (\beta x)[A+\exp (\beta x)]^{-1} .
$$

The constant $A$ is given by the boundary condition. By comparison of Eqs. (AB) and (A7) the solution for Eq. (A7) is

$$
\Delta Q_{t}=-(1 / d) \exp \left(-b_{t} t\right)\left[A+\exp \left(-b_{t} t\right)\right]^{-1} \text {. }
$$

With the boundary condition $\Delta Q_{t}(0)=\Delta Q_{10}$ the parameter $A$ is found:

$$
\begin{aligned}
& \Delta Q_{t 0}=-1 /[(1+A) d], \\
& A=-\left[1+1 /\left(d \Delta Q_{t 0}\right] .\right.
\end{aligned}
$$

Insertion of $A$ in Eq. (A9) yields the final result:

$$
\begin{aligned}
\Delta Q_{t}(t)= & \frac{1}{d} \exp \left(-b_{t} t\right) \\
& \times\left(1+\frac{1}{\left(d \Delta Q_{10}\right)}-\exp \left(-b_{t} r\right)\right)^{-1} .
\end{aligned}
$$

On a long time scale the exponential term in the denomisator is negligible, so that the decay of $\Delta Q_{t}$ is given by an exponential time constant $\tau_{i}^{\text {dec }}$ :

$$
\tau_{t}^{\mathrm{dec}}=\left(b_{t}\right)^{-1}=\left(v_{\mathrm{tht}} \sigma_{t}^{\mathrm{th}} n_{\mathrm{gb}}\right)^{-1}
$$

The rise of the photosignal is initially cominated by the excitation of occupied deep trap states. The recombination of holes into the shallow states giver by an increase of $\Delta Q$, occurs with some time delay with respect to the increase of $\Delta Q_{t}$ so that the assumption $\Delta Q_{s}(t) \ll \Delta Q_{t}(t)$ is valid, at least for a short time, after the light is switched on with a photon fux density $F_{0}$. In this case the differential equation (A4) reads as follows: 
$\frac{\partial \Delta Q_{i}}{\partial t}=-b_{i} d\left(\Delta Q_{t}\right)^{2}-\left(b_{i}+\sigma_{t}^{\mathrm{opt}} k F_{0}\right) \Delta Q_{t}+\sigma_{t}^{\mathrm{op} i} k F_{0} Q_{t}$

This ordinary differential equation is of the form

$$
\frac{\partial y}{\partial x}=\alpha y^{2}+\beta y+\gamma
$$

with the general solution:

$$
y(x)=-\frac{\Gamma_{+} \exp \left(\Gamma_{+} x\right)+A \Gamma_{-} \exp \left(\Gamma_{-} x\right)}{\alpha\left[\exp \left(\Gamma_{+} x\right)+A \exp \left(\Gamma_{-}\right)\right]}
$$

where $\Gamma_{+},-$is given by

$$
\Gamma_{+,-}=\frac{1}{2}\left(\beta \pm \sqrt{\beta^{2}-4 \alpha \gamma}\right) \text {. }
$$

By comparison of Eq. (A12) with (A11) the constants $\alpha, \beta$, and $\gamma$ are given as a function of $d, b_{t}, \sigma_{s}^{\text {opt }}$, and $F_{0}$. The parameter $A$ follows from the boundary condition $\Delta Q_{t}(0)=0$.

$A=\frac{\sqrt{\left(b_{t}+\sigma_{i}^{o p t} k F_{0}\right)^{2}+4 d Q_{i} b_{i} \sigma_{i}^{o p t} k F_{0}}-\left(b_{t}+\sigma_{t}^{o p t} k F_{0}\right)}{\sqrt{\left(b_{i}+\sigma_{i}^{o p t} k F_{0}\right)^{2}+4 d Q_{i} b_{i} \sigma_{i}^{o p t} k E_{0}}+\left(b_{t}+\sigma_{t}^{o p} k F_{0}\right)}$.

with some transformation $\Delta Q_{r}(t)$ is finally given by

$$
\begin{aligned}
\Delta Q_{t}(t)= & -\frac{1}{2 d b_{t}}\left(\frac{1}{\tau_{t}^{\text {att }}}-b_{t}-\sigma_{t}^{\text {apt }} k F\right) \\
& \times \frac{1-\exp \left(-t / \tau_{t}^{\text {att }}\right)}{1+A \exp \left(-t / \tau_{t}^{\text {att }}\right)},
\end{aligned}
$$

where the time constant $\tau_{i}^{\text {att }}$ is defined by

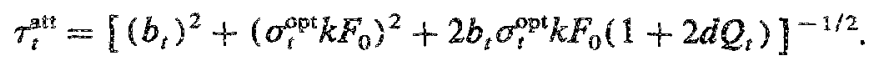

From the definition of $d$ [see Eq. (A1)] it can be seen that $d Q_{t} \leqslant 1$ holds. According to the results of measurement and by use of Eq. (A10) the parameter $b_{t}$ is estimated to be of the order of $10^{6} \mathrm{~s}^{-1}$, so that the factor $A$ is much smaller than one. Using this estimation for Eq. (A14) the initial increase of $\Delta Q_{t}$ is proportional to $\left[1-\exp \left(-t / \tau_{t}^{\text {tt }}\right)\right]$ being characterized by an exponential increase with the time constant $\tau_{i}^{\text {att }}$

The thermal capture cross section $\sigma_{s}^{\text {th }}$ for the shallow trap states is expected to be larger than the cross section for the deep trap states. This implies that the recombination will for the first time be dominated by the variation of $\Delta Q_{s}$ while $\Delta Q_{t}$ is approximately constant and equal to $\Delta Q_{t 0}$. The differential equation (A5) is again of the form of Eq. (A.12) with $\alpha, \beta$, and $\gamma$ given by

$$
\begin{aligned}
& \alpha=d b_{t} \\
& \beta=-b_{i}\left(C+d \Delta Q_{i o}\right) \\
& \gamma=b_{i} d Q_{s}^{u} \Delta Q_{t o}
\end{aligned}
$$

With the boundary condition $\Delta Q_{s}(0)=\Delta Q_{\text {so }}$ the solution is found in the same way as shown above.

$$
\Delta Q_{s}(n)=\frac{\Xi Q_{s 0} \cosh \left(t b_{t} \Xi / 2\right)-\left[2 d Q_{s}^{n} \Delta Q_{t 0}+\left(C+d \Delta Q_{t 0}\right) \Delta Q_{s 0}\right] \sinh \left(t b_{t} \Xi / 2\right)}{\Xi \cosh \left(t b_{t} \Xi / 2\right)+\left(C+d \Delta Q_{t 0}+2 d \Delta Q_{s 0}\right) \sinh \left(t b_{t} \Xi / 2\right)}
$$

with

$$
\Xi=\left[\left(C+d \Delta Q_{t 0}\right)^{2}-4 d^{2} Q_{s}^{u} \Delta Q_{t o}\right]^{1 / 2}
$$

Bearing in mind that

$$
C>1, \quad \Delta Q_{s 0}<\Delta Q_{t 0}<Q_{t} \leq 1 / d, \quad d Q_{s}^{u} \leqslant 1,
$$

the parameter $\Xi$ is about $C$ and $E q$. (A17) can be approximated to

$$
\Delta Q_{s}(t)=\frac{\Delta Q_{s 0}\left[\cosh \left(t / 2 \tau_{s}^{\mathrm{dec}}\right)-\sinh \left(t / 2 \tau_{s}^{\mathrm{dec}}\right)\right]-2 d Q_{s}^{*} \Delta Q_{s 0} / C \sinh \left(t / 2 \tau_{s}^{\mathrm{dec}}\right)}{\cosh \left(t / 2 \tau_{s}^{\mathrm{dec}}\right)+\sinh \left(t / 2 \tau_{s}^{\mathrm{dec}}\right)},
$$

immediately yielding

$$
\begin{aligned}
\Delta Q_{s}(t)= & \Delta Q_{s D} \exp \left(-t / \tau_{s}^{\text {dec }}\right) \\
& -d Q_{s}^{u} \Delta Q_{t 0} / C\left[1-\exp \left(-t / \tau_{s}^{\mathrm{dec}}\right)\right],
\end{aligned}
$$

and finally

$$
\begin{aligned}
\Delta Q_{s}(t)= & \left(\Delta Q_{s 0}+d Q_{s}^{u} \Delta Q_{t} / C\right) \exp \left(-t / \tau_{s}^{d e u}\right) \\
& -d Q_{s}^{u} \Delta Q_{t 0} / C
\end{aligned}
$$

where

$$
\tau_{s}^{\text {dee }}=\left(b_{i} C\right)^{-1}=\left[b_{t}\left(1+d Q_{s}^{u}+Q_{s}^{k} / Q_{s}^{o}\right)\right]^{-1} .
$$

The time-dependent second term in Eq. (A19) has its origin in the fact that $\Delta Q_{s}(t)$ is not zero as long as $\Delta Q_{t}(t)$ has a final value. In the applied model the shallow trap level represents the extersion of the band-tail states in the grains into the grain boundary, so that the time dependence of the recombination into the shallow traps can be taken from the majority carrier lifetime $\tau_{p}=\tau_{s}^{\text {dec }}$ given by the transient interband photosignal. 
${ }^{1}$ N. C. -C. Lus L. Gerzberg, C.-Y. La, and J. D. Meind, IEEE Trans. Electron Devices ED-28, 818 (1981).

${ }^{2}$ H. Schaber, D. Curter, J. Binder, and E. Obermier, J. Appl. Phys. 58,4633 (1983).

3J. Manoliu and T. I. Kamins, Soldd-State Electron. 15, 1103 (1972).

${ }^{4}$ D. B. Meakin, P. A. Coxon, P. Migliorato, J. Stoemenos, and N. A. Economou, Appl. Phys. Lett. 50, 1894 (1987).

${ }^{5}$ M. Feisi and A. W. Wieder, IEEE Trans. Electron Devices ED-30, 1792 (1983).

${ }^{6}$ J. Y. W. Seto, J. Appl. Phys. 46,5247 (1975).

${ }^{7}$ G. E. Pike and C. H. Seager, J. Appl. Phys. 50, 3414 (1979).

${ }^{8}$ J. Werner and M. Peisl, Phys. Rev. B 31, 6881 (1985).

${ }^{9}$ M. S. Bennett, I. Appl. Phys. 58, 3470 (1985).

${ }^{10}$ A. Rose, Concepts in Photocanductivity and Allied Problems (Krieger,
New York, 1978).

${ }^{11}$ C. H. Sezger and G. E. Pike, Appl. Phys. Lett. 40, 471 (1982).

${ }^{12}$ W. Bock and W. Prettl, Appl. Phys. Lett. 53, 19 (1988).

${ }^{13}$ J. Werner, in Polycrystalline Semiconductors, edited by G. Harbeke (Springer, New York, 1985).

${ }^{14}$ H. C. De Graaff, M. Huybers, and J. G. DeGroot, Solid-State Electron. 25, 67 (1982).

${ }^{15}$ D. - L. Chen, D. W. Greve, and A. M. Guzman, IEEE Trans. Elextron Devices ED-35, 1045 (1988).

${ }^{16}$ D. P. Joshi and R. S. Srivastava, J. Appl. Phys. 56, 2375 (1984).

${ }^{17}$ E. Poon, E. S. Yang, H. L. Evans, W. Hwang, and R. M. Osgood, Jr., Appl. Phys. Lett. 42,285 (1983).

${ }^{18}$ W. B. Jackson, N. M. Johnson, and D. K. Biegelsen, Appl. Phys. Lett. 43, $195(1983)$. 